\title{
O SON DA NOSA AULA: UNA EXPERIENCIA ORQUESTAL EN TIEMPOS DE CONFINAMIENTO
}

\author{
Chao-Fernández, Rocío ${ }^{1}$; Rosa Napal, Francisco César ${ }^{2}$; Chao-Fernández, Aurelio ${ }^{3}$ \\ ${ }^{1}$ Universidade da Coruña, Facultade de Ciencias da Educación \\ ORCID 0000-0002-6584-0049 \\ 2 Universidade da Coruña, Facultade de Ciencias da Educación \\ ORCID 0000-0003-2916-8707 \\ ${ }^{3}$ Universidade da Coruña, Facultade de Ciencias da Educación \\ ORCID 0000-0001-7846-7637
}

\section{RESUMEN}

Las limitaciones impuestas por el COVID 19 afectaron negativamente al desarrollo de las asignaturas de carácter práctico, en este caso la expresión musical, por lo que fue necesario buscar alternativas tecnológicas que permitieran la realización de actividades musicales colectivas. Esta experiencia se inició en el itinerario de Música del Máster universitario en profesorado de educación secundaria obligatoria, bachillerato, formación profesional y enseñanza de idiomas, dentro de la materia Proyectos de Innovación e Investigación Educativa en Música, siendo la primera vez que este itinerario se impartía en la UDC. Con el confinamiento, el trabajo final tomó forma de concierto, para demostrar que, aún desde el aislamiento, el aula de música seguía trabajando. A medida que el proyecto se conformaba, se fueron incorporando estudiantes de doctorado, que llevan varios años haciendo de mentores de alumnado de Música del Máster en Didácticas Específicas, así como el alumnado de Grado. El primer reto fue la ejecución de una obra orquestal estando cada intérprete en su domicilio. Para garantizar el cumplimiento del objetivo general, se estableció un organigrama y un cronograma con los repartos de roles y las diferentes fases de realización. Independientemente de algunas dificultades técnicas inevitables, los resultados superaron las expectativas iniciales, al recibir el reconocimiento de la comunidad universitaria, de la prensa y de los medios de comunicación.

PALABRAS CLAVE: expresión musical; TIC; trabajo colectivo; confinamiento 


\section{CITA RECOMENDADA:}

Chao Fernández, Rocío; Rosa Napal, Francisco César; Chao Fernández, Aurelio (2021): 0 son da nosa aula: una experiencia orquestal en tiempos de confinamiento. En García Naya, J.A. (ed.) (2021). Contextos universitarios transformadores: a nova normalidade académica. Leccións aprendidas e retos de futuro. V Xornadas de Innovación Docente. Cufie. Universidade da Coruña. A Coruña (pág.81-93). DOI capítulo: https://doi.org/10.17979/spudc.9788497498180.081

DOl libro: https://doi.org/10.17979/spudc.9788497498180

\section{ABSTRACT}

The limitations imposed by COVID 19 negatively affected the development of practical subjects, in this case, Musical Expression, which made it necessary to look for technological alternatives that would allow for collective musical activities. This experience began in the Music itinerary of the Master's Degree in Secondary Education, within the subject Projects of Educational Innovation and Research in Music, being the first time that this itinerary was taught at the UDC. Due to lockdown, the final assignment was to perform in a concert to prove that, even in isolation, the music classroom continued to work. As the project took shape, both doctoral students (who have been mentoring Music students in the Master's Degree in Specific Didactics for several years) and undergraduate students joined the project. The first challenge was the performance of an orchestral work while each performer was at their own home. In order to guarantee the fulfillment of the general objective, an organizational chart and a chronogram were established with the distribution of roles and the different phases of realization. Despite some unavoidable technical difficulties, the results exceeded initial expectations, receiving recognition from the university community, the press, and the media.

KEY WORDS: musical expression; ICT; collective work; confinement 


\section{INTRODUCCIÓN}

Esta experiencia se llevó a cabo en el curso 2019-2020 en la Facultad de Ciencias de la Educación de la Universidad de A Coruña. En medio de la crisis sanitaria provocada por el COVID 19, las asignaturas prácticas propias del Itinerario de Música que forman parte del Máster Universitario en Profesorado de Educación Secundaria Obligatoria y Bachillerato, Formación Profesional y Enseñanza de Idiomas tuvieron que cambiar de la modalidad presencial a la virtual. En la signatura Proyectos de Innovación e Investigación educativa en Música, surgió la iniciativa de continuar trabajando de forma colectiva, a pesar de las restricciones impuestas por la pandemia en cuanto al aislamiento personal.

El empleo de las TIC fue uno de los elementos determinantes en esta decisión, ya que otra de las asignaturas presentes en el Itinerario de Música trata, precisamente, del empleo de las tecnologías aplicadas a la producción y a la educación musical. Tras explorar las posibilidades técnicas existentes para lograr un resultado aceptable, comenzó el proceso de planificación del proyecto cuyos objetivos generales fueron:

- Continuar, pese a las limitaciones, el trabajo musical colectivo a través de las TIC en el módulo de Música.

- Comprobar si es posible la docencia musical online en su vertiente de práctica colectiva.

- Acompañar emocionalmente a la sociedad en su confinamiento desde una perspectiva artística.

Las personas participantes fueron - por parte del alumnado- dieciséis estudiantes del Máster, siete de Doctorado y dos de Grado en Educación Primaria. Por su parte, el profesorado estuvo representado por los tres miembros del área de Didáctica de la Expresión Musical, sumando un total de veinticinco integrantes.

Desde el punto de vista temporal, el trabajo fue dividido en diferentes fases: selección de la obra a trabajar; contacto con las autoridades encargadas de los derechos de autor; realización 
de la orquestación específica contando con los medios instrumentales; grabación individual de cada músico y, finalmente, el montaje global audiovisual.

Una vez realizado el trabajo en su totalidad, se pudo llegar a una serie de conclusiones sobre la manera en las que se cumplió el propósito general del proyecto, sobre las dificultades encontradas en el proceso y sobre las nuevas propuestas que se pudieran derivar de esta.

\section{DESCRIPCIÓN DE LA EXPERIENCIA}

\subsection{FUNDAMENTACIÓN TEÓRICA}

Cualquier producción audiovisual requiere una organización interna que, debido a la práctica en el tiempo, se ha estandarizado. Según Soto (2013, p. 123), "este tipo de actividad se puede separar en tres etapas fundamentales: la preproducción, la producción y la postproducción". Las tres etapas pueden estar articuladas por fases más precisas, siempre dependiendo da las características específicas de la producción en sí, y adaptándose a cualquier situación excepcional, como pueden ser las restricciones de movilidad y de reunión que impuso el COVID 19.

Teniendo todo esto en cuenta, se desprende de aquí que, para acometer un proyecto como este se debe contar con una separación de roles, pero al mismo tiempo con una gran coherencia en cuanto al trabajo en equipo. Sobre esta dualidad, Ortega-Lupiáñez (2015) afirma que "lo arduo de la tarea es conseguir un equilibrio donde cada miembro del grupo sienta que su trabajo es relevante" (p. 41).

En el caso de una producción audiovisual en la que la música es protagonista y en la que van a participar numerosos intérpretes, es importante asumir un cuidado extra en el mantenimiento de las normas establecidas, tanto desde el punto de vista específicamente musical como desde el cuidado de la imagen. Es importante recalcar en este punto que, al tratarse de la confluencia de dos tipos de lenguaje, deben observarse las teorizaciones surgidas a partir de los estudios realizados sobre los medios audiovisuales. En este sentido, Alcalde (2008), 
empleando el término oído audiovisual, se refiere al nuevo significado que adquieren la música y la imagen cuando coinciden en un único producto artístico.

Por otra parte, uno de los retos de la actualidad es el manejo cada vez más especializado de las tecnologías en cualquiera de las áreas de conocimiento y en cualquier actividad profesional. En el caso de la música y de la educación musical, las herramientas de las que se dispone son múltiples, entre las que se pueden distinguir los secuenciadores MIDI, cuya función es "registrar, modificar y reproducir secuencias de eventos musicales, funcionando con un formato de varias pistas en las que es posible grabar mensajes digitales de manera independiente." (Rosa-Napal, 2017, p. 92). Otro grupo de aplicaciones está constituido por los editores de partituras, cuyas ventajas son numerosas ya que permiten la escritura musical con una gran calidad gráfica y además brinda la posibilidad de escuchar lo que se ha escrito. Por último, los editores de audio — destinados a la grabación y a la edición de sonido— resultan ser herramientas imprescindibles en la práctica y en la educación musical en la actualidad (Chao-Fernández, Pérez Crego y Chao-Fernández).

\subsection{FASES DE REALIZACIÓN}

Como se ha expresado anteriormente, este proyecto contó con diferentes fases en el proceso de producción, así como en la organización de los roles (Figura 1). Seguidamente se mostrará cada una de ellas con los detalles pertinentes en cada caso. Es importante señalar que, tanto el cronograma como el organigrama, están establecidos de una manera lineal, aunque, en el transcurso de la práctica, fueron necesarias algunas modificaciones impuestas por las necesidades que fueron apareciendo en el proceso.

Al dividirse en cinco fases, en el diseño del trabajo se ha tenido en consideración que estas estén contenidas cronológicamente en las tres grandes etapas propuestas por Soto (2013), por lo que la primera y la segunda fase quedarían incluidas en la etapa de preproducción, la tercera y la cuarta formarían parte de la producción y la quinta constituiría la etapa de postproducción. 


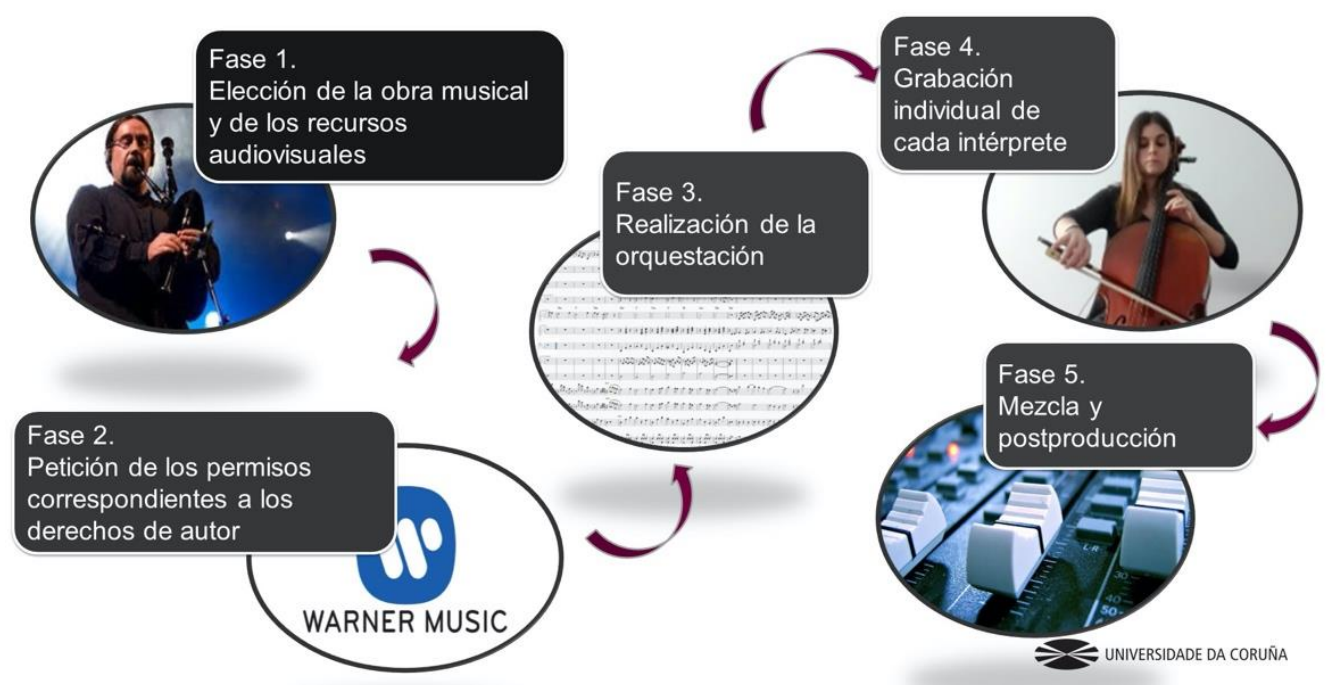

Figura 1. Fases de la realización. Nota. Elaboración propia

La lista de roles y de personas, constituye el organigrama del proyecto. En la Figura 2, es posible observar las diferentes funciones desarrolladas durante el proceso por el equipo. Es necesario destacar aquí que dichos roles fueron asumidos en su totalidad por las personas participantes, por lo que, como se puede comprobar, hay integrantes que realizaron más de una tarea. Al finalizar el vídeo se presentaron los correspondientes créditos, tal y como es habitual en las propuestas audiovisuales. 
Diseñoy gestión Rocío Chao Fernández Aurelio Chao Fernández

\section{Organización:}

Rocío Chao Fernández

Aurelio Chao Fernández

Francisco César Rosa Napal

Abraham Felpeto Guerrero

Rubén Vázquez Sánchez
Autor de la obra

Bieito Romero

Orquestación

Francisco César Rosa Napal

Montaje de audio y producción

Abraham Felpeto Guerrero

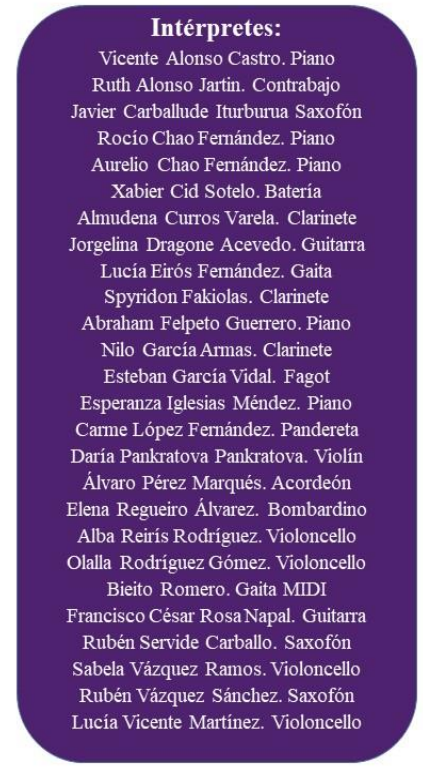

Figura 2. Organigrama del proyecto. Nota. Elaboración propia

\subsubsection{Fase I. Elección de la obra musical y de los recursos audiovisuales a emplear}

En primer lugar, tras el análisis de la situación sanitaria que provocó cambios radicales en la forma en que se imparten las materias prácticas, el profesorado del Itinerario de Música del Máster Universitario en Profesorado de Educación Secundaria Obligatoria y Bachillerato, Formación Profesional y Enseñanza de Idiomas, independientemente de las diferentes asignaturas que se imparten en dicho módulo, coincidió en que en la materia Proyectos de Innovación e Investigación Educativa en Música era posible llevar a cabo una intervención como la que se ha descrito con anterioridad.

El criterio de selección unánimemente consensuado indicaba que la pieza no debería tener grandes dificultades técnicas en cuanto a la interpretación instrumental, ya que cada intérprete podría resolver los problemas técnicos de forma individual.

Otros factores de estos criterios fueron, por una parte, su presencia en los currículos oficiales de los diferentes niveles educativos en las asignaturas relacionadas con la expresión musical $y$, por otra, el significado cultural que pudiera representar para las personas de la Comunidad 
Autónoma de Galicia. El resultado de la elección fue, de manera unánime, la melodía de la pieza instrumental 0 son do ar, cuyo autor es el compositor gallego Bieito Romero, quien también participó como intérprete en el proyecto.

\subsubsection{Fase II. Petición de los permisos correspondientes a los derechos de autor}

Siempre que se emplean materiales sonoros -0 de cualquier tipo- que han sido publicados o registrados oficialmente con anterioridad, es imprescindible contar con la autorización de las personas 0 de las entidades que salvaguardan la autoría de las obras. En este caso, la obra instrumental elegida, 0 son do ar, forma parte del disco homónimo grabado en 1988 por el grupo gallego Luar na Lubre y versionada en 1996 por Mike Oldfield en su álbum The Voyager, cuyos derechos de autor son actualmente administrados por la productora Warner Music.

En esta segunda fase se solicitaron dichos permisos, por lo que fue necesario contactar directamente con la productora siguiendo todos los trámites pertinentes, lo que supuso una difícil negociación en cuanto a los derechos de explotación y, sobre todo, al tiempo de exposición concedido para la utilización de los temas melódicos de la obra elegida. Esta limitación temporal influyó en la estructura formal que adoptaría la versión final de la orquestación.

\subsubsection{Fase III. Realización de la orquestación}

Una vez obtenidos los correspondientes permisos, se procedió a la orquestación, condicionada por la instrumentación disponible y contando con la participación de todas las posibilidades instrumentales procedentes del alumnado y de la totalidad del profesorado del módulo de Música.

El primer paso de esta fase fue el recuento de los distintos instrumentos disponibles y su organización en diferentes secciones de acuerdo a su naturaleza organológica, lo que dio lugar a un formato heterogéneo y no común en las formaciones orquestales establecidas por los estándares de la música académica, del que formaron parte: un violín, cuatro violonchelos, un contrabajo, dos guitarras y cinco pianos, en la familia de las cuerdas. Entre los instrumentos 
aerófonos se pudo contar —en la familia de viento madera — con un acordeón, dos gaitas, tres saxofones, un fagot y tres clarinetes, mientras que en viento metal participó un bombardino. Finalmente, la formación instrumental se completó con la sección de percusión, en la que participaron una batería estándar y una pandereta. La configuración final del formato quedó establecida tanto en secciones como en subfamilias instrumentales: cuerdas frotadas, cuerdas pulsadas y cuerdas percutidas; instrumentos de viento madera y de viento metal e instrumentos de percusión.

Teniendo en cuenta la gran riqueza tímbrica disponible, se procedió a elaborar una partitura orquestal en la que cada sección tuvo un papel destacado para conseguir la mayor variedad sonora posible.

\subsubsection{Fase IV. Grabación individual de cada intérprete}

En esta fase se realizó el envío de las partes individuales (particelli) a cada intérprete editadas con la aplicación MuseScore, al mismo tiempo que se redactó un documento en el que se indicaban todos los pasos a seguir en el proceso de grabación. Con estas normas se pretendía que tanto el sonido como la imagen tuvieran una coherencia y una calidad aceptables con vistas a facilitar el trabajo de la siguiente fase de mezcla y postproducción. También se estableció la manera de entregar las grabaciones individuales en una carpeta común creada para concentrar todas las muestras en una cuenta de OneDrive.

Para lograr una perfecta sincronización, se realizó la exportación de la partitura orquestal a un archivo MIDI, mediante el cual sería posible escuchar íntegramente a todos los instrumentos en el momento de la grabación. Este archivo se puso a disposición de los instrumentistas con el propósito de que cada intérprete se sintiera parte del conjunto y, al mismo tiempo, arropado por el resto de músicos. Como medio de garantizar la exactitud del tempo, se dotó al archivo MIDI de una claqueta, empleando posteriormente el editor de audio Audacity para su exportación final al tipo de archivo mp3. 
Las grabaciones fueron realizadas por cada uno de los integrantes -en sus respectivos domicilios - con sus teléfonos móviles y enviadas según el formato establecido, aunque en algunos casos, al no ajustarse a las indicaciones iniciales, tuvieron que repetirse varias veces hasta lograr la calidad deseada.

\subsubsection{Fase V. Mezcla y postproducción}

Tras haber obtenido y revisado todo el material audiovisual aportado por cada participante, comenzó el proceso de montaje del audio. La aplicación disponible para esa tarea fue Presonus Studio One, un secuenciador virtual que permite ecualizar pistas de audio y de MIDI de manera individual para luego mezclarlas y finalmente llegar a la masterización total de la dimensión sonora.

En relación a la imagen, se tuvo en cuenta la gran variedad de escenarios obtenidos para crear una composición coherente, en la que se buscó en todo momento el equilibrio visual partiendo de la unión dialéctica de la analogía y el contraste. En vez de emplear un fondo de pantalla común a todos los intérpretes, se decidió que se pudieran observar las imágenes de los domicilios de cada uno, buscando así un mensaje de cercanía cálida dentro del aislamiento. El software editor de vídeo que se empleó para el correspondiente montaje fue Adobe Premiere, ya que es una aplicación con funciones compatibles con otros programas y que, por otra parte, permite obtener un producto final de gran calidad.

Para dar por terminada la fase de postproducción, se realizaron varias visualizaciones y audiciones de las mezclas, corrigiendo cualquier detalle sobre todo en la ecualización del sonido.

\section{RESULTADOS}

El resultado final estuvo representado por un vídeo de 2,06 minutos de duración, en una pantalla múltiple dividida en veintisiete paneles, uno para cada intérprete más la identificación institucional (Figura 3). Es posible tener acceso al vídeo en la página web de la Facultad de 
Ciencias da Educación de la UDC, en la sección Media 0 a través del siguiente enlace: https://www.educacion.udc.es/index.php?pagina=expresion-musical

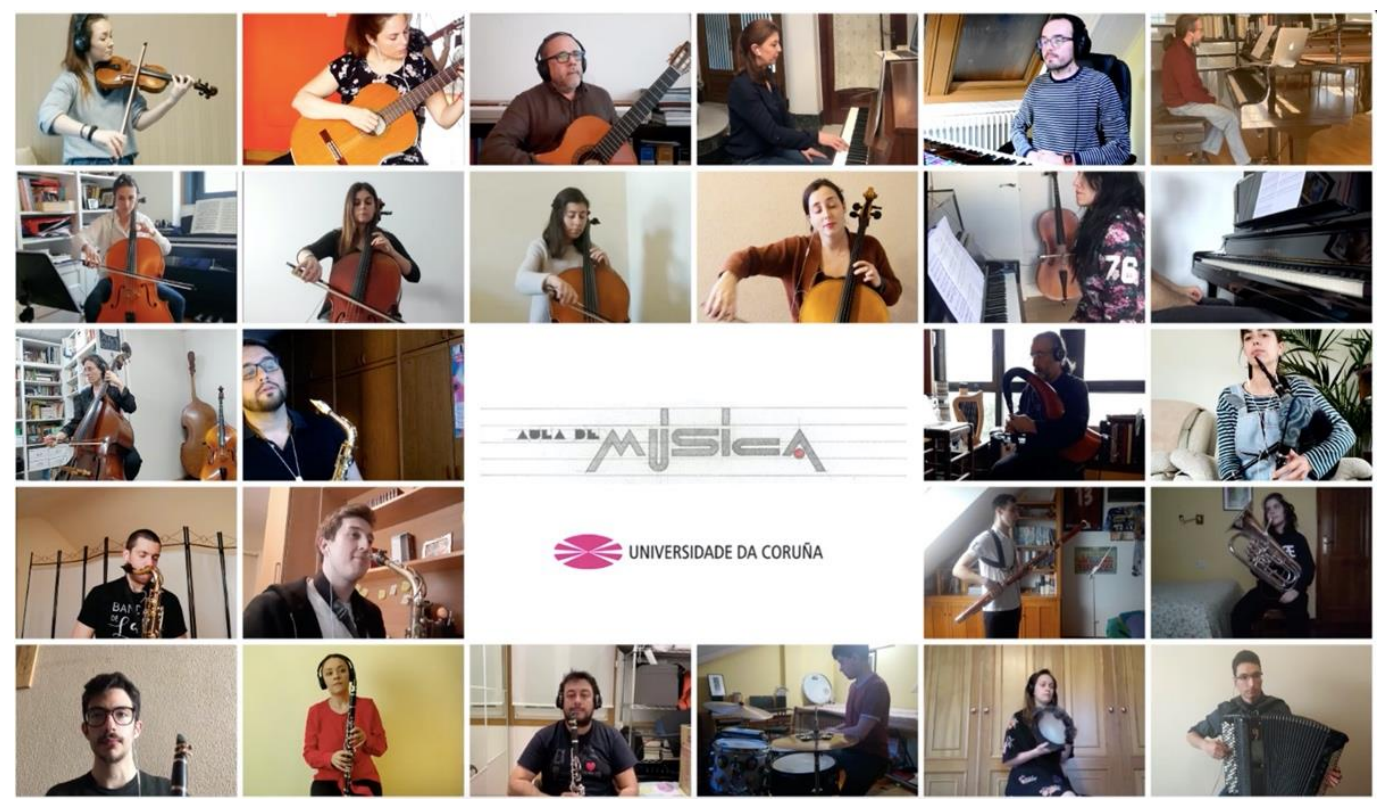

Figura 3. Pantalla múltiple. Nota. Imagen del proyecto audiovisual 0 son da nosa aula.

Luego de difundir el trabajo a través de diferentes medios, se recibieron varios mensajes de agradecimiento y de reconocimiento como los siguientes, los cuales se citan en el idioma original en el que fueron redactados y recibidos:

- Moitas gracias polo fermoso agasallo desa peza musical. A todo o alumnado implicado e a quen impulsáchedes a iniciativa. Sen dúbida 0 arte está facendo mellores os nosos días.

- Quero unir a miña voz ao coro de loanzas con que os compañeiros acolleron a vosa iniciativa. Nunca foi máis verdadeira a sentencia que lemos no célebre cadro de Vermeer: Musica laetitiae comes, medicina dolorum.

- Felicidades por vuestro proyecto e idea. La música siempre es necesaria y bienvenida. 


\section{CONCLUSIONES}

A modo de reflexión final, se recogen en este apartado las principales conclusiones a las que se llegó después de haber analizado cada uno de los factores que formaron parte del proyecto desde su inicio hasta su culminación:

- Los objetivos propuestos fueron conseguidos en su totalidad, incluso es posible afirmar que la implicación de cada miembro del equipo superó las expectativas iniciales.

- Se pudo comprobar que es posible trabajar contenidos musicales colectivos en condiciones de aislamiento físico.

- La propuesta tuvo una buena acogida por el alumnado, el profesorado de la comunidad educativa universitaria y el público en general, cumpliendo con el objetivo de enviar un mensaje musical cálido en medio de un confinamiento nunca deseado.

- Las limitaciones dadas por las condiciones técnicas estuvieron ocasionadas por el delay (retaso de la señal sonora) en las videollamadas; la baja calidad de sonido en algunas muestras, debido a la capacidad de cada uno de los dispositivos empleados individualmente y los problemas de conexión o infraestructura, entre otros. No obstante, mediante el empeño del colectivo, pudieron ser subsanadas satisfactoriamente.

- Debido a los resultados positivos obtenidos, tanto en el proceso de elaboración como en los resultados finales y constatado el impacto de este tipo de trabajo en el clima de trabajo en grupo, a partir de este pequeño proyecto podrían derivarse otros con múltiples enfoques y combinaciones transversales que podrían materializarse en investigaciones 0 en nuevas propuestas didácticas.

\section{REFERENCIAS}

Alcalde, J. (2008). La interacción música-imagen en la cultura de la escucha. En M. de Aguilera, J. Adell \& A. Sedeño (eds.), Comunicación y música I. Lenguaje y medios (pp. 141-160). Editorial UOC. 
Chao-Fernández, A., Pérez Crego, Mª C. y Chao-Fernández, R. (2020). La grabación musical como herramienta de aprendizaje. Implicaciones educativas para el alumnado. Revista de estudios e Investigación en Psicología y Educación, 7(1), 71-83. https://doi.org/10.17979/reipe.2020.7.1.6520

Chao-Fernández, R.; Vázquez-Sánchez, R. y Felpeto-Guerrero, A. (2020). Audacity como herramienta para la creación de materiales educativos. Una aproximación a través del MOOC "Música para el siglo XXl". Revista Latinoamericana de Tecnología Educativa, 19(1), 121137. http://dx.doi.org/10.17398/1695-288X.19.1.121

Ortega-Lupiáñez, C, (2015). La producción audiovisual en el aula, Magazin 23, 40-46.

Rosa-Napal, F. (2017). Guía de referencia para Didáctica de la Expresión Musical. Repronor.

Soto, J. (2013). Estandarización de organigramas y modelamiento del proceso de producción audiovisual: una propuesta basada en la toma de decisiones Cuadernos.info, 33, 121-131. DOl: 10.7764/cdi.33.525 
